\title{
Grüss type integral inequalities for generalized Riemann-Liouville $k$-fractional integrals
}

\section{Shahid Mubeen* and Sana lqbal}

"Correspondence:

smjhanda@gmail.com

Department of Mathematics,

University of Sargodha, Sargodha,

Pakistan

\begin{abstract}
Integral inequalities are considered to be important as they have many applications described by a number of researchers. Moreover, the theory of fractional calculus is used in solving differential, integral, and integro-differential equations and also in various other problems involving special functions. In this research article, we present the improved version of generalizations for a Grüss type integral inequality by taking a generalized Riemann-Liouville fractional integral in terms of a new parameter $k>0$. We contribute in the on going research by providing mathematical results that can be verified easily.
\end{abstract}

Keywords: Grüss inequality; Riemann-Liouville fractional integral; Pochhammar k-symbol; gamma k-function; inequalities

\section{Introduction}

Since the integral inequalities are considered to be important, therefore the research has been proceeded to extend the investigation for such type of inequalities. Such inequalities and its applications are extensively described in many articles (see, for instance, [1-5]). Among such types of inequalities, Grüss inequality is considered to be more interesting, perhaps. Grüss inequality could be defined as follows.

Definition 1 Let $f, g:[a, b] \rightarrow \mathbb{R}$ be integrable functions such that

$$
\varphi<f(x)<\Phi \text { and } \quad \psi<g(x)<\Psi \quad \text { for all } x \in[a, b] .
$$

Then

$$
\begin{aligned}
& \left|\frac{1}{b-a} \int_{a}^{b} f(x) g(x) d x-\frac{1}{(b-a)^{2}} \int_{a}^{b} f(x) d x \int_{a}^{b} g(x) d x\right| \\
& \quad \leq \frac{1}{4}(\Phi-\varphi)(\Psi-\psi),
\end{aligned}
$$

where the constant $\frac{1}{4}$ is sharp and $\varphi, \Phi, \psi, \Psi \in \mathbb{R}$.

The integral inequality (1) (Grüss inequality) as described above, actually connects the integral of the product of two functions with the product of their integrals.

(c) 2016 Mubeen and labal. This article is distributed under the terms of the Creative Commons Attribution 4.0 International License (http://creativecommons.org/licenses/by/4.0/), which permits unrestricted use, distribution, and reproduction in any medium, provided you give appropriate credit to the original author(s) and the source, provide a link to the Creative Commons license, and indicate if changes were made. 
Definition 2 A function $f(t)$ is said to be in $L_{p, r}[a, b]$ if

$$
\left(\int_{a}^{b}|f(t)|^{p} t^{r} d t\right)^{\frac{1}{p}}<\infty, \quad 1 \leq p<\infty, r \geq 0 .
$$

Definition 3 If $f \in L_{1}[a, b]$. Then Riemann-Liouville fractional integral of order $\alpha \geq 0$ is defined by

$$
I_{a}^{\alpha} f(t)=\frac{1}{\Gamma(\alpha)} \int_{a}^{t}(t-x)^{\alpha-1} f(x) d x, \quad t \in[a, b],
$$

where $\Gamma$ is the Euler gamma function.

Definition 4 If $f \in L_{1, r}[a, b]$, then the generalized Riemann-Liouville fractional integral $I_{a}^{\alpha, r}$ of order $\alpha \geq 0$ and $r \geq 0$, introduced by is defined by

$$
I_{a}^{\alpha, r} f(t)=\frac{(r+1)^{1-\alpha}}{\Gamma(\alpha)} \int_{a}^{t}\left(t^{r+1}-x^{r+1}\right)^{\alpha-1} x^{r} f(x) d x, \quad t \in[a, b]
$$

where $\Gamma$ is the Euler gamma function.

Kaçar and Yildirim [6] studied Grüss type inequality and provided useful results. Due to the importance of such results and applications (see [6]), we study the existing results and provide noval results. We introduce parameter $k>0$ and generalize the results in such a way that the existing results can be explored too. Thus, the results provided in this research paper are more generalized as compared to the existing results.

\subsection{Applications}

Inequalities involving functions of two or more independent variables play fundamental role in the continuous development of the theory, methods and applications of differential and integral equations. In view of the wider applications, integral inequalities have received considerable attention. Recently, different versions of such inequalities have been developed which are useful in the study of different classes of differential and integral equations. These inequalities act as ready tools to study the classes of differential and integral equations [7].

It is well known that Grüss type inequalities in continuous and discrete cases play a crucial role in studying the qualitative behavior of differential and difference equations, respectively, as well as many other areas of mathematics. Motivated by Grüss [8], our purpose is to prove more general versions of Grüss type inequalities.

\subsection{Related work}

Researchers focused in investigating such integrals and provided remarkable results for inequalities involving Riemann-Liouville fractional integrals like the Grüss, Chebyshev and Hermite-Hadamard type inequalities for integrable functions as well as for convex functions (see [9-15]).

Recently, Diaz and Pariguan [16] have defined the generalization of the classical gamma and beta functions in terms of a new parameter $k>0$, called gamma and beta $k$-functions, 
respectively:

$$
\Gamma_{k}(x)=\lim _{n \rightarrow \infty} \frac{n ! k^{n}(n k)^{\frac{x}{k}-1}}{(x)_{n, k}}, \quad k>0, x \in \mathbb{C} \backslash k Z^{-},
$$

where

$$
(x)_{n, k}=x(x+k)(x+2 k) \cdots(x+(n-1) k), \quad n \geq 1,
$$

is called the Pochhammer $k$-symbol. The integral representation of the gamma $k$-function is

$$
\Gamma_{k}(x)=\int_{0}^{\infty} t^{x-1} e^{-\frac{t^{k}}{k}} d t, \quad \operatorname{Re}(x)>0 .
$$

In 2010, Krasniqi [17] proved some inequalities and monotonicity for the ratio of gamma $k$-function. Later, in 2012, using the above definitions, Mubeen and Habibullah [18] have introduced the $k$-fractional integral of the Riemann-Liouville type as follows:

$$
I_{a, k}^{\alpha} f(t)=\frac{1}{k \Gamma_{k}(\alpha)} \int_{a}^{t}(t-x)^{\frac{\alpha}{k}-1} f(x) d x, \quad t \in[a, b]
$$

where $\Gamma_{k}$ is the Euler gamma $k$-function.

In 2013, Kokologiannaki and Krasniqi [19] gave completely monotonicity properties and inequalities for functions involving the gamma and psi $k$-functions. They also introduced the $k$-analogue of the Riemann Zeta function and obtained some inequalities relating gamma and zeta $k$-functions. Romero et al. [20] introduced a new fractional operator called the $k$-Riemann-Liouville fractional derivative by using gamma $k$-function. They also proved some properties of this newly defined fractional operator and found its relationship with the Riemann-Liouville $k$-fractional integral.

During the past few years, many researchers investigated a large number of inequalities involving the fractional $q$-integral operators. In 2014, Baleanu and Agarwal [21] established some inequalities involving Saigo fractional $q$-integral operator in the theory of quantum calculus by using the two parameters of deformation. They also presented the corresponding inequalities involving Riemann-Liouville and Kober fractional $q$-integral operators respectively as special cases. Choi and Agarwal [22] established some new Saigo type fractional integral inequalities and their $q$-analogue. As their special cases, they proved the corresponding inequalities involving Riemann-Liouville and Erdélyi-Kober type fractional integral operators.

Agarwal et al. [23] proved some new fractional integral inequalities involving generalized Erdélyi-Kober fractional $q$-integral operator. They also considered the cases of synchronous functions as well as the functions bounded by integrable functions. Sarikaya and Karaca [24] gave a generalization of the Riemann-Liouville $k$-fractional integral with some properties. Also they proved some new integral inequalities involving this generalized Riemann-Liouville $k$-fractional integral.

In 2015, Choi et al. [25] established some new inequalities involving generalized ErdélyiKober fractional $q$-integral operator of the two parameters of deformation. Liu et al. [26] proved some new integral inequalities of Gronwall-Bellman-Bihari type with delay for 
discontinuous functions which generalize and improve some former famous results about inequalities and which is helpful to discuss the qualitative and quantitative properties for solutions to some nonlinear differential and integral equations.

Sarikaya et al. [27] have introduced $(k, r)$-fractional integral of the Riemann type as

$$
I_{a, k}^{\alpha, r} f(t)=\frac{(r+1)^{1-\frac{\alpha}{k}}}{k \Gamma_{k}(\alpha)} \int_{a}^{t}\left(t^{r+1}-x^{r+1}\right)^{\frac{\alpha}{k}-1} x^{r} f(x) d x, \quad t \in[a, b],
$$

where $\Gamma_{k}$ is the Euler gamma $k$-function.

\section{Results and discussion}

In this section, we prove some Grüss type inequalities involving the generalized RiemannLiouville $k$-fractional integral $I_{a, k}^{\alpha, r}$ defined in (2).

Theorem 2.1 For $k>0$, let $f \in L_{1, r}[a, b]$ and $r \geq 0, \alpha, \beta>0$. Suppose that there exist two integrable functions $\varphi_{1}, \varphi_{2}$ on $[a, b]$ such that

$$
\varphi_{1}(t) \leq f(t) \leq \varphi_{2}(t), \quad \forall t \in[a, b] .
$$

Then the inequality

$$
\begin{aligned}
& I_{a, k}^{\beta, r} \varphi_{1}(t) I_{a, k}^{\alpha, r} f(t)+I_{a, k}^{\alpha, r} \varphi_{2}(t) I_{a, k}^{\beta, r} f(t) \\
& \quad \geq I_{a, k}^{\alpha, r} \varphi_{2}(t) I_{a, k}^{\beta, r} \varphi_{1}(t)+I_{a, k}^{\alpha, r} f(t) I_{a, k}^{\beta, r} f(t)
\end{aligned}
$$

holds true.

Proof From inequality (3), for all $x, y \in[a, b]$, we have

$$
\left(\varphi_{2}(x)-f(x)\right)\left(f(y)-\varphi_{1}(y)\right) \geq 0 .
$$

This implies that

$$
\varphi_{2}(x) f(y)+\varphi_{1}(y) f(x) \geq \varphi_{1}(y) \varphi_{2}(x)+f(x) f(y) .
$$

By multiplying both sides of above inequality with

$$
\frac{(r+1)^{2-\frac{\alpha}{k}-\frac{\beta}{k}}\left(t^{r+1}-x^{r+1}\right)^{\frac{\alpha}{k}-1}\left(t^{r+1}-y^{r+1}\right)^{\frac{\beta}{k}-1} x^{r} y^{r}}{k^{2} \Gamma_{k}(\alpha) \Gamma_{k}(\beta)}
$$

and then integrating it with respect to $x$ and $y$ from $a$ to $t$, we obtain the required inequality.

Lemma 2.2 Put $r=0$ in Theorem 2.1, we get

$$
I_{a, k}^{\beta} \varphi_{1}(t) I_{a, k}^{\alpha} f(t)+I_{a, k}^{\alpha} \varphi_{2}(t) I_{a, k}^{\beta} f(t) \geq I_{a, k}^{\alpha} \varphi_{2}(t) I_{a, k}^{\beta} \varphi_{1}(t)+I_{a, k}^{\alpha} f(t) I_{a, k}^{\beta} f(t) .
$$

Theorem 2.3 For $k>0$, let $f \in L_{1, r}[a, b]$. Suppose that $m \leq f(t) \leq M$, for all $t \in[a, b]$ and $m, M \in \mathbb{R}$. Then, for $r \geq 0, \alpha, \beta>0$, we have 


$$
\begin{aligned}
& M \frac{(r+1)^{\frac{-\alpha}{k}} t^{(r+1) \frac{\alpha}{k}}}{\Gamma_{k}(\alpha+k)} I_{a, k}^{\beta, r} f(t)+m \frac{(r+1)^{\frac{-\beta}{k}} t^{(r+1) \frac{\beta}{k}}}{\Gamma_{k}(\beta+k)} I_{a, k}^{\alpha, r} \\
& \quad \geq m M \frac{(r+1)^{-\frac{(\alpha+\beta)}{k}} t^{(r+1)\left(\frac{\alpha+\beta}{k}\right)}}{\Gamma_{k}(\alpha+k) \Gamma_{k}(\beta+k)}+I_{a, k}^{\alpha, r} f(t) I_{a, k}^{\beta, r} f(t) .
\end{aligned}
$$

Proof Since $m \leq f(t) \leq M$ for all $t \in[a, b]$, for all $x, y \in[a, b]$, we have

$$
(M-f(x))(f(y)-m) \geq 0 .
$$

The required inequality can be proved by using the above inequality and following the steps of Theorem 2.1.

Lemma 2.4 Put $r=0$ in Theorem 2.3, we get

$$
\begin{aligned}
& M \frac{t^{\frac{\alpha}{k}}}{\Gamma_{k}(\alpha+k)} I_{a, k}^{\beta} f(t)+m \frac{t^{\frac{\beta}{k}}}{\Gamma_{k}(\beta+k)} I_{a, k}^{\alpha} f(t) \\
& \quad \geq m M \frac{t^{\frac{\alpha+\beta}{k}}}{\Gamma_{k}(\alpha+k) \Gamma_{k}(\beta+k)}+I_{a, k}^{\alpha} f(t) I_{a}, k^{\beta} f(t) .
\end{aligned}
$$

Theorem 2.5 For $k>0$, let $f$ and $g$ be two integrable functions on $[a, b]$ and $r \geq 0, \alpha, \beta>0$. Suppose that (3) holds and, moreover, assume that there exist integrable functions $\psi_{1}$ and $\psi_{2}$ on $[a, b]$ such that

$$
\psi_{1}(t) \leq g(t) \leq \psi_{2}(t), \quad \forall t \in[a, b]
$$

Then the following inequalities hold:

(i) $I_{a, k}^{\beta, r} \psi_{1}(t) I_{a, k}^{\alpha, r} f(t)+I_{a, k}^{\alpha, r} \varphi_{2}(t) I_{a, k}^{\beta, r} g(t) \geq I_{a, k}^{\beta, r} \psi_{1}(t) I_{a, k}^{\alpha, r} \varphi_{2}(t)+I_{a, k}^{\alpha, r} f(t) I_{a, k}^{\beta, r} g(t)$,

(ii) $I_{a, k}^{\beta, r} \varphi_{1}(t) I_{a, k}^{\alpha, r} g(t)+I_{a, k}^{\alpha, r} \psi_{2}(t) I_{a, k}^{\beta, r} f(t) \geq I_{a, k}^{\beta, r} \varphi_{1}(t) I_{a, k}^{\alpha, r} \psi_{2}(t)+I_{a, k}^{\alpha, r} g(t) I_{a, k}^{\beta, r} f(t)$,

(iii) $I_{a, k}^{\alpha, r} \varphi_{2}(t) I_{a, k}^{\beta, r} \psi_{2}(t)+I_{a, k}^{\alpha, r} f(t) I_{a, k}^{\beta, r} g(t) \geq I_{a, k}^{\alpha, r} \varphi_{2}(t) I_{a, k}^{\beta, r} g(t)+I_{a, k}^{\beta, r} \psi_{2}(t) I_{a, k}^{\alpha, r} f(t)$,

(iv) $I_{a, k}^{\alpha, r} \varphi_{1}(t) I_{a, k}^{\beta, r} \psi_{1}(t)+I_{a, k}^{\alpha, r} f(t) I_{a, k}^{\beta, r} g(t) \geq I_{a, k}^{\alpha, r} \varphi_{1}(t) I_{a, k}^{\beta, r} g(t)+I_{a, k}^{\beta, r} \psi_{1}(t) I_{a, k}^{\alpha, r} f(t)$.

Proof (i): From (3) and (4) for all $t \in[a, b]$, we have

$$
\left(\varphi_{2}(x)-f(x)\right)\left(g(y)-\psi_{1}(y)\right) \geq 0
$$

then

$$
\varphi_{2}(x) g(y)+\psi_{1}(y) f(x) \geq \psi_{1}(y) \varphi_{2}(x)+f(x) g(y) .
$$

By multiplying both sides of above inequality with

$$
\frac{(r+1)^{2-\frac{\alpha}{k}-\frac{\beta}{k}}\left(t^{r+1}-x^{r+1}\right)^{\frac{\alpha}{k}-1}\left(t^{r+1}-y^{r+1}\right)^{\frac{\beta}{k}-1} x^{r} y^{r}}{k^{2} \Gamma_{k}(\alpha) \Gamma_{k}(\beta)}
$$

and then integrating with respect to $x$ and $y$ over $(a, t)$, we obtain (i).

To prove (ii)-(iv), we use the following inequalities

(ii) $(\psi(x)-g(x))\left(f(y)-\varphi_{1}(y)\right) \geq 0$, 
(iii) $\left(\varphi_{2}(x)-f(x)\right)\left(g(y)-\psi_{2}(y)\right) \leq 0$,

(iv) $\left(\varphi_{1}(x)-f(x)\right)\left(g(y)-\psi_{1}(y)\right) \leq 0$.

By following the steps of the above theorem, the following lemma can be proved.

Lemma 2.6 For $k>0$, let $f$ and $g$ be two integrable functions on $[a, b]$ and $r \geq 0, \alpha, \beta>0$. Suppose that there exist $m, M, n, N \in \mathbb{R}$ such that

$$
m \leq f(t) \leq M, \quad n \leq g(t) \leq N, \quad \forall t \in[a, b]
$$

Then we have

$\left(\mathrm{i}^{*}\right) \quad n \frac{(r+1)^{\frac{-\beta}{k}} t^{(r+1) \frac{\beta}{k}}}{\Gamma_{k}(\beta+k)} I_{a, k}^{\alpha, r} f(t)+M \frac{(r+1)^{\frac{-\alpha}{k}} t^{(r+1) \frac{\alpha}{k}}}{\Gamma_{k}(\alpha+k)} I_{a, k}^{\beta, r} g(t)$

$$
\geq n M \frac{(r+1)^{-\frac{\alpha+\beta}{k}} t^{(r+1) \frac{\alpha+\beta}{k}}}{\Gamma_{k}(\alpha+k) \Gamma_{k}(\beta+k)}+I_{a, k}^{\alpha, r} f(t) I_{a, k}^{\beta, r} g(t),
$$

$\left(\mathrm{ii}^{*}\right) \quad m \frac{(r+1)^{\frac{-\beta}{k}} t^{(r+1) \frac{\beta}{k}}}{\Gamma_{k}(\beta+k)} I_{a, k}^{\alpha, r} g(t)+N \frac{(r+1)^{\frac{-\alpha}{k}} t^{(r+1) \frac{\alpha}{k}}}{\Gamma_{k}(\alpha+k)} I_{a, k}^{\beta, r} f(t)$

$$
\geq m N \frac{(r+1)^{-\frac{\alpha+\beta}{k}} t^{(r+1) \frac{\alpha+\beta}{k}}}{\Gamma_{k}(\alpha+k) \Gamma_{k}(\beta+k)}+I_{a, k}^{\beta, r} f(t) I_{a, k}^{\alpha, r} g(t),
$$

$\left(\mathrm{iii}^{*}\right) \quad M \frac{(r+1)^{\frac{-\alpha}{k}} t^{(r+1) \frac{\alpha}{k}}}{\Gamma_{k}(\alpha+k)} I_{a, k}^{\beta, r} g(t)+N \frac{(r+1)^{\frac{-\beta}{k}} t^{(r+1) \frac{\beta}{k}}}{\Gamma_{k}(\beta+k)} I_{a, k}^{\alpha, r} f(t)$

$$
\leq M N \frac{(r+1)^{-\frac{\alpha+\beta}{k}} t^{(r+1) \frac{\alpha+\beta}{k}}}{\Gamma_{k}(\alpha+k) \Gamma_{k}(\beta+k)}+I_{a, k}^{\alpha, r} f(t) I_{a, k}^{\beta, r} g(t),
$$

$\left(\mathrm{iv}^{*}\right) \quad m \frac{(r+1)^{\frac{-\alpha}{k}} t^{(r+1) \frac{\alpha}{k}}}{\Gamma_{k}(\alpha+k)} I_{a, k}^{\beta, r} g(t)+n \frac{(r+1)^{\frac{-\beta}{k}} t^{(r+1) \frac{\beta}{k}}}{\Gamma_{k}(\beta+k)} I_{a, k}^{\alpha, r} f(t)$

$$
\leq m n \frac{(r+1)^{-\frac{\alpha+\beta}{k}} t^{(r+1) \frac{\alpha+\beta}{k}}}{\Gamma_{k}(\alpha+k) \Gamma_{k}(\beta+k)}+I_{a, k}^{\alpha, r} f(t) I_{a, k}^{\beta, r} g(t) .
$$

Theorem 2.7 For $k>0$, let $f \in L_{1, r}[a, b]$ and let $\varphi_{1}, \varphi_{2}$ be two integrable functions on $[a, b]$ and $r \geq 0, \alpha>0$. Suppose that condition (3) holds. Then

$$
\begin{aligned}
& \frac{(r+1)^{\frac{-\alpha}{k}} t^{(r+1) \frac{\alpha}{k}}}{\Gamma_{k}(\alpha+k)} I_{a, k}^{\alpha, r} f^{2}(t)-\left[I_{a, k}^{\alpha, r} f(t)\right]^{2} \\
& =\left(I_{a, k}^{\alpha, r} \varphi_{2}(t)-I_{a, k}^{\alpha, r} f(t)\right)\left(I_{a, k}^{\alpha, r} f(t)-I_{a, k}^{\alpha, r} \varphi_{1}(t)\right) \\
& \quad-\frac{(r+1)^{\frac{-\alpha}{k}} t^{(r+1) \frac{\alpha}{k}}}{\Gamma_{k}(\alpha+k)}\left(I_{a, k}^{\alpha, r} \varphi_{2}(t)-I_{a, k}^{\alpha, r} f(t)\right)\left(I_{a, k}^{\alpha, r} f(t)-I_{a, k}^{\alpha, r} \varphi_{1}(t)\right) \\
& +\frac{(r+1)^{\frac{-\alpha}{k}} t^{(r+1) \frac{\alpha}{k}}}{\Gamma_{k}(\alpha+k)} I_{a, k}^{\alpha, r}\left(\varphi_{1}(t) f(t)\right)-I_{a, k}^{\alpha, r} \varphi_{1}(t) I_{a, k}^{\alpha, r} f(t) \\
& +\frac{(r+1)^{\frac{-\alpha}{k}} t^{(r+1) \frac{\alpha}{k}}}{\Gamma_{k}(\alpha+k)} I_{a, k}^{\alpha, r}\left(\varphi_{2}(t) f(t)\right)-I_{a, k}^{\alpha, r} \varphi_{2}(t) I_{a, k}^{\alpha, r} f(t) \\
& \quad-\frac{(r+1)^{\frac{-\alpha}{k}} t^{(r+1) \frac{\alpha}{k}}}{\Gamma_{k}(\alpha+k)} I_{a, k}^{\alpha, r}\left(\varphi_{1}(t) \varphi_{2}(t)\right)+I_{a, k}^{\alpha, r} \varphi_{1}(t) I_{a, k}^{\alpha, r} \varphi_{2}(t) .
\end{aligned}
$$


Proof For any $x, y \in[a, b]$ and $r \geq 0$, we have

$$
\begin{aligned}
\left(\varphi_{2}(y)\right. & -f(y))\left(f(x)-\varphi_{1}(x)\right)+\left(\varphi_{2}(x)-f(x)\right)\left(f(y)-\varphi_{1}(y)\right) \\
& -\left(\varphi_{2}(x)-f(x)\right)\left(f(x)-\varphi_{1}(x)\right)-\left(\varphi_{2}(y)-f(y)\right)\left(f(y)-\varphi_{1}(y)\right) \\
= & f^{2}(x)+f^{2}(y)-2 f(x) f(y)+\varphi_{2}(y) f(x)+\varphi_{1}(x) f(y) \\
& -\varphi_{1}(x) \varphi_{2}(y)+\varphi_{2}(x) f(y)+\varphi_{1}(y) f(x)-\varphi_{1}(y) \varphi_{2}(y) \\
& -\varphi_{2}(x) f(x)+\varphi_{1}(x) \varphi_{2}(x)-\varphi_{1}(x) f(x)-\varphi_{2}(y) f(y) \\
& +\varphi_{1}(y) \varphi_{2}(y)-\varphi_{1}(y) f(y) \\
\Rightarrow & \\
\left(\varphi_{2}(y)\right. & -f(y))\left(I_{a, k}^{\alpha, r} f(t)-I_{a, k}^{\alpha, r} \varphi_{1}(t)\right)+\left(I_{a, k}^{\alpha, r} \varphi_{2}(t)-I_{a, k}^{\alpha, r} f(t)\right)\left(f(y)-\varphi_{1}(y)\right) \\
& -I_{a, k}^{\alpha, r}\left[\left(\varphi_{2}(t)-f(t)\right)\left(f(t)-\varphi_{1}(t)\right)\right]-\left(\varphi_{2}(y)-f(y)\right)\left(f(y)-\varphi_{1}(y)\right) \frac{(r+1)^{\frac{-\alpha}{k}} t^{(r+1) \frac{\alpha}{k}}}{\Gamma_{k}(\alpha+k)} \\
= & I_{a, k}^{\alpha, r} f^{2}(t)+f^{2}(y) \frac{(r+1)^{\frac{-\alpha}{k}} t^{(r+1) \frac{\alpha}{k}}}{\Gamma_{k}(\alpha+k)}-2 f(y) I_{a, k}^{\alpha, r} f(t)+\varphi_{2}(y) I_{a, k}^{\alpha, r} f(t)+\varphi_{2}(y) I_{a, k}^{\alpha, r} \varphi_{1}(t) \\
& +f(y) I_{a, k}^{\alpha, r} \varphi_{2}(t)+f(y) I_{a, k}^{\alpha, r} \varphi_{1}(t)+\varphi_{1}(y) I_{a, k}^{\alpha, r} f(t)-\varphi_{1}(y) I_{a, k}^{\alpha, r} \varphi_{2}(t)-I_{a, k}^{\alpha, r}\left(\varphi_{2}(t) f(t)\right) \\
& +I_{a, k}^{\alpha, r}\left(\varphi_{1}(t) \varphi_{2}(t)\right)-I_{a, k}^{\alpha, r}\left(\varphi_{1}(t) f(t)\right)-\varphi_{2}(y) f(y) \frac{(r+1)^{\frac{-\alpha}{k}} t^{(r+1) \frac{\alpha}{k}}}{\Gamma_{k}(\alpha+k)} \\
& +\varphi_{1}(y) \varphi_{2}(y) \frac{(r+1)^{\frac{-\alpha}{k}} t^{(r+1) \frac{\alpha}{k}}}{\Gamma_{k}(\alpha+k)}-\varphi_{1}(y) f(y) \frac{(r+1)^{\frac{-\alpha}{k}} t^{(r+1) \frac{\alpha}{k}}}{\Gamma_{k}(\alpha+k)} .
\end{aligned}
$$

Multiplying both sides of the above equation by

$$
\frac{(r+1)^{1-\frac{\beta}{k}}\left(t^{r+1}-y^{r+1}\right)^{\frac{\beta}{k}-1} y^{r}}{k \Gamma_{k}(\beta)}
$$

and integrating with respect to $y$ over $(a, t)$, we obtain the required equality (5).

Lemma 2.8 For $k>0$, let $r=0$ in Theorem 2.7, we get

$$
\begin{aligned}
& \frac{t^{\frac{\alpha}{k}}}{\Gamma_{k}(\alpha+k)} I_{a, k}^{\alpha} f^{2}(t)-\left[I_{a, k}^{\alpha} f(t)\right]^{2} \\
& =\left(I_{a, k}^{\alpha} \varphi_{2}(t)-I_{a, k}^{\alpha} f(t)\right)\left(I_{a, k}^{\alpha} f(t)-I_{a, k}^{\alpha} \varphi_{1}(t)\right) \\
& \quad-\frac{t^{\frac{\alpha}{k}}}{\Gamma_{k}(\alpha+k)}\left(I_{a, k}^{\alpha} \varphi_{2}(t)-I_{a, k}^{\alpha} f(t)\right)\left(I_{a, k}^{\alpha} f(t)-I_{a, k}^{\alpha} \varphi_{1}(t)\right) \\
& \quad+\frac{t^{\frac{\alpha}{k}}}{\Gamma_{k}(\alpha+k)} I_{a, k}^{\alpha}\left(\varphi_{1}(t) f(t)\right)-I_{a, k}^{\alpha} \varphi_{1}(t) I_{a, k}^{\alpha} f(t) \\
& \quad+\frac{t^{\frac{\alpha}{k}}}{\Gamma_{k}(\alpha+k)} I_{a, k}^{\alpha}\left(\varphi_{2}(t) f(t)\right)-I_{a, k}^{\alpha} \varphi_{2}(t) I_{a, k}^{\alpha} f(t) \\
& \quad-\frac{t^{\frac{\alpha}{k}}}{\Gamma_{k}(\alpha+k)} I_{a, k}^{\alpha}\left(\varphi_{1}(t) \varphi_{2}(t)\right)+I_{a, k}^{\alpha} \varphi_{1}(t) I_{a, k}^{\alpha} \varphi_{2}(t) .
\end{aligned}
$$


Corollary 2.9 For $k>0$, let $f \in[a, b]$. Suppose that $m \leq f(t) \leq M$ for all $t \in[a, b]$ and $m, M \in \mathbb{R}$. Then, for $r \geq 0$ and $\alpha>0$, we have

$$
\begin{aligned}
& \frac{(r+1)^{\frac{-\alpha}{k}} t^{(r+1) \frac{\alpha}{k}}}{\Gamma_{k}(\alpha+k)} I_{a, k}^{\alpha, r} f^{2}(t)-\left[I_{a, k}^{\alpha, r} f(t)\right]^{2} \\
& =-\frac{(r+1)^{\frac{-\alpha}{k}} t^{(r+1) \frac{\alpha}{k}}}{\Gamma_{k}(\alpha+k)} I_{a, k}^{\alpha, r}[(M-f(t))(f(t)-m)] \\
& \quad+\left[\frac{M(r+1)^{\frac{-\alpha}{k}} t^{(r+1) \frac{\alpha}{k}}}{\Gamma_{k}(\alpha+k)}-I_{a, k}^{\alpha, r} f(t)\right] \\
& \quad \times\left[I_{a, k}^{\alpha, r} f(t)-\frac{m(r+1)^{\frac{-\alpha}{k}} t^{(r+1) \frac{\alpha}{k}}}{\Gamma_{k}(\alpha+k)}\right] .
\end{aligned}
$$

Proof This equality can be proved by using $\varphi_{1}(t)=m$ and $\varphi_{2}(t)=M$ in Theorem 2.7.

Theorem 2.10 For $k>0$, let $f$ and $g$ be two integrable functions on $[a, b]$ and let $\varphi_{1}, \varphi_{2}$, $\psi_{1}$, and $\psi_{2}$ be four integrable functions satisfying the conditions (3) and (4) on $[a, b]$. Then for all $t \in[a, b], r \geq 0$, and $\alpha>0$

$$
\begin{aligned}
& \left|\frac{(r+1)^{\frac{-\alpha}{k}} t^{(r+1) \frac{\alpha}{k}}}{\Gamma_{k}(\alpha+k)} I_{a, k}^{\alpha, r}(f(t) g(t))-I_{a, k}^{\alpha, r} f(t) I_{a, k}^{\alpha, r} g(t)\right| \\
& \quad \leq \sqrt{S_{k}^{r}\left(f, \varphi_{1}, \varphi_{2}\right) S_{k}^{r}\left(g, \psi_{1}, \psi_{2}\right)},
\end{aligned}
$$

where

$$
\begin{aligned}
S_{k}^{r}(x, y, z)= & \left(I_{a, k}^{\alpha, r} z(t)-I_{a, k}^{\alpha, r} x(t)\right)\left(I_{a, k}^{\alpha, r} x(t)-I_{a, k}^{\alpha, r} y(t)\right) \\
& +\frac{(r+1)^{\frac{-\alpha}{k}} t^{(r+1) \frac{\alpha}{k}}}{\Gamma_{k}(\alpha+k)} I_{a, k}^{\alpha, r}(y(t) x(t))-I_{a, k}^{\alpha, r} y(t) I_{a, k}^{\alpha, r} x(t) \\
& +\frac{(r+1)^{\frac{-\alpha}{k}} t^{(r+1) \frac{\alpha}{k}}}{\Gamma_{k}(\alpha+k)} I_{a, k}^{\alpha, r}(z(t) x(t))-I_{a, k}^{\alpha, r} z(t) I_{a, k}^{\alpha, r} x(t) \\
& -\frac{(r+1)^{\frac{-\alpha}{k}} t^{(r+1) \frac{\alpha}{k}}}{\Gamma_{k}(\alpha+k)} I_{a, k}^{\alpha, r}(y(t) z(t))+I_{a, k}^{\alpha, r} y(t) I_{a, k}^{\alpha, r} z(t) .
\end{aligned}
$$

Proof Since $f$ and $g$ are two integrable functions on $[a, b]$ and satisfy the conditions (3) and (4), we define

$$
\begin{aligned}
& B(x, y)=[f(x)-f(y)][g(x)-g(y)] \\
& \quad=f(x) g(x)+f(y) g(y)-f(x) g(y)-f(y) g(x) \\
& \Rightarrow \quad \\
& \frac{(r+1)^{2-\frac{2 \alpha}{k}}}{2 k^{2} \Gamma_{k}^{2}(\alpha)} \int_{a}^{t} \int_{a}^{t}\left(t^{r+1}-x^{r+1}\right)^{\frac{\alpha}{k}-1}\left(t^{r+1}-y^{r+1}\right)^{\frac{\alpha}{k}-1} x^{r} y^{r} B(x, y) d x d y \\
& \quad=\frac{(r+1)^{\frac{-\alpha}{k}} t^{(r+1) \frac{\alpha}{k}}}{\Gamma_{k}(\alpha+k)} I_{a, k}^{\alpha, r}(f(t) g(t))-I_{a, k}^{\alpha, r} f(t) I_{r, k}^{\alpha} g(t) .
\end{aligned}
$$


Now by using the value of $B(x, y)$ from (7) to the left hand side of equality (8) and then applying the Cauchy-Schwarz inequality, we have

$$
\begin{aligned}
& \left(\frac{(r+1)^{2-\frac{2 \alpha}{k}}}{2 k^{2} \Gamma_{k}^{2}(\alpha)} \int_{a}^{t} \int_{a}^{t}\left(t^{r+1}-x^{r+1}\right)^{\frac{\alpha}{k}-1}\left(t^{r+1}-y^{r+1}\right)^{\frac{\alpha}{k}-1} x^{r} y^{r} B(x, y) d x d y\right)^{2} \\
& \leq \frac{(r+1)^{2-\frac{2 \alpha}{k}}}{2 k^{2} \Gamma_{k}^{2}(\alpha)} \int_{a}^{t} \int_{a}^{t}\left(t^{r+1}-x^{r+1}\right)^{\frac{\alpha}{k}-1}\left(t^{r+1}-y^{r+1}\right)^{\frac{\alpha}{k}-1} x^{r} y^{r}[f(x)-f(y)]^{2} d x d y \\
& \quad \times \frac{(r+1)^{2-\frac{2 \alpha}{k}}}{2 k^{2} \Gamma_{k}^{2}(\alpha)} \int_{a}^{t} \int_{a}^{t}\left(t^{r+1}-x^{r+1}\right)^{\frac{\alpha}{k}-1}\left(t^{r+1}-y^{r+1}\right)^{\frac{\alpha}{k}-1} x^{r} y^{r}[g(x)-g(y)]^{2} d x d y .
\end{aligned}
$$

Now since

$$
[f(x)-f(y)]^{2}=f^{2}(x)+f^{2}(y)-2 f(x) f(y)
$$

one can easily prove that

$$
\begin{aligned}
& \frac{(r+1)^{2-\frac{2 \alpha}{k}}}{2 k^{2} \Gamma_{k}^{2}(\alpha)} \int_{a}^{t} \int_{a}^{t}\left(t^{r+1}-x^{r+1}\right)^{\frac{\alpha}{k}-1}\left(t^{r+1}-y^{r+1}\right)^{\frac{\alpha}{k}-1} x^{r} y^{r}[f(x)-f(y)]^{2} d x d y \\
& \quad=\frac{(r+1)^{\frac{-\alpha}{k}} t^{(r+1) \frac{\alpha}{k}}}{\Gamma_{k}(\alpha+k)} I_{a, k}^{\alpha, r} f^{2}(t)-\left(I_{a, k}^{\alpha, r} f(t)\right)^{2} .
\end{aligned}
$$

Similarly,

$$
\begin{aligned}
& \frac{(r+1)^{2-\frac{2 \alpha}{k}}}{2 k^{2} \Gamma_{k}^{2}(\alpha)} \int_{a}^{t} \int_{a}^{t}\left(t^{r+1}-x^{r+1}\right)^{\frac{\alpha}{k}-1}\left(t^{r+1}-y^{r+1}\right)^{\frac{\alpha}{k}-1} x^{r} y^{r}[g(x)-g(y)]^{2} d x d y \\
& \quad=\frac{(r+1)^{\frac{-\alpha}{k}} t^{(r+1) \frac{\alpha}{k}}}{\Gamma_{k}(\alpha+k)} I_{a, k}^{\alpha, r} g^{2}(t)-\left(I_{a, k}^{\alpha, r} g(t)\right)^{2} .
\end{aligned}
$$

Using equations (10) and (11) into (9), we get

$$
\begin{aligned}
& \left(\frac{(r+1)^{2-\frac{2 \alpha}{k}}}{2 k^{2} \Gamma_{k}^{2}(\alpha)} \int_{a}^{t} \int_{a}^{t}\left(t^{r+1}-x^{r+1}\right)^{\frac{\alpha}{k}-1}\left(t^{r+1}-y^{r+1}\right)^{\frac{\alpha}{k}-1} x^{r} y^{r} B(x, y) d x d y\right)^{2} \\
& \leq\left[\frac{(r+1)^{\frac{-\alpha}{k}} t^{(r+1) \frac{\alpha}{k}}}{\Gamma_{k}(\alpha+k)} I_{a, k}^{\alpha, r} f^{2}(t)-\left(I_{a, k}^{\alpha, r} f(t)\right)^{2}\right] \\
& \quad \times\left[\frac{(r+1)^{\frac{-\alpha}{k}} t^{(r+1) \frac{\alpha}{k}}}{\Gamma_{k}(\alpha+k)} I_{a, k}^{\alpha, r} g^{2}(t)-\left(I_{a, k}^{\alpha, r} g(t)\right)^{2}\right] .
\end{aligned}
$$

Thus the equation (8) together with the inequality (12) implies that

$$
\begin{gathered}
\left(\frac{(r+1)^{\frac{-\alpha}{k}} t^{(r+1) \frac{\alpha}{k}}}{\Gamma_{k}(\alpha+k)} I_{a, k}^{\alpha, r}(f(t) g(t))-I_{a, k}^{\alpha, r} f(t) I_{a, k}^{\alpha, r} g(t)\right)^{2} \\
\leq\left[\frac{(r+1)^{\frac{-\alpha}{k}} t^{(r+1) \frac{\alpha}{k}}}{\Gamma_{k}(\alpha+k)} I_{a, k}^{\alpha, r} f^{2}(t)-\left(I_{a, k}^{\alpha, r} f(t)\right)^{2}\right] \\
\quad \times\left[\frac{(r+1)^{\frac{-\alpha}{k}} t^{(r+1) \frac{\alpha}{k}}}{\Gamma_{k}(\alpha+k)} I_{a, k}^{\alpha, r} g^{2}(t)-\left(I_{a, k}^{\alpha, r} g(t)\right)^{2}\right] .
\end{gathered}
$$


Now since

$$
\left(\varphi_{2}(t)-f(t)\right)\left(f(t)-\varphi_{1}(t)\right) \geq 0
$$

and

$$
\left(\psi_{2}(t)-g(t)\right)\left(g(t)-\phi_{1}(t)\right) \geq 0,
$$

therefore,

$$
\frac{(r+1)^{\frac{-\alpha}{k}} t^{(r+1) \frac{\alpha}{k}}}{\Gamma_{k}(\alpha+k)} I_{a, k}^{\alpha, r}\left(\varphi_{2}(t)-f(t)\right)\left(f(t)-\varphi_{1}(t)\right) \geq 0, \quad t \in[a, b]
$$

and

$$
\frac{(r+1)^{\frac{-\alpha}{k}} t^{(r+1) \frac{\alpha}{k}}}{\Gamma_{k}(\alpha+k)} I_{a, k}^{\alpha, r}\left(\varphi_{2}(t)-f(t)\right)\left(f(t)-\varphi_{1}(t)\right) \geq 0, \quad t \in[a, b] .
$$

By Theorem 2.7, we have

$$
\begin{aligned}
& \frac{(r+1)^{\frac{-\alpha}{k}} t^{(r+1) \frac{\alpha}{k}}}{\Gamma_{k}(\alpha+k)} I_{a, k}^{\alpha, r} f^{2}(t)-\left[I_{a, k}^{\alpha, r} f(t)\right]^{2} \\
& \leq\left(I_{a, k}^{\alpha, r} \varphi_{2}(t)-I_{a, k}^{\alpha, r} f(t)\right)\left(I_{a, k}^{\alpha, r} f(t)-I_{a, k}^{\alpha, r} \varphi_{1}(t)\right) \\
& \quad+\frac{(r+1)^{\frac{-\alpha}{k}} t^{(r+1) \frac{\alpha}{k}}}{\Gamma_{k}(\alpha+k)} I_{a, k}^{\alpha, r}\left(\varphi_{1}(t) f(t)\right)-I_{a, k}^{\alpha, r} \varphi_{1}(t) I_{a, k}^{\alpha, r} f(t) \\
& \quad+\frac{(r+1)^{\frac{-\alpha}{k}} t^{(r+1) \frac{\alpha}{k}}}{\Gamma_{k}(\alpha+k)} I_{a, k}^{\alpha, r}\left(\varphi_{2}(t) f(t)\right)-I_{a, k}^{\alpha, r} \varphi_{2}(t) I_{a, k}^{\alpha, r} f(t) \\
& \quad-\frac{(r+1)^{\frac{-\alpha}{k}} t^{(r+1) \frac{\alpha}{k}}}{\Gamma_{k}(\alpha+k)} I_{a, k}^{\alpha, r}\left(\varphi_{1}(t) \varphi_{2}(t)\right)+I_{a, k}^{\alpha, r} \varphi_{1}(t) I_{a, k}^{\alpha, r} \varphi_{2}(t) \\
& =S_{k}^{r}\left(f, \varphi_{1}, \varphi_{2}\right) .
\end{aligned}
$$

Similarly,

$$
\begin{aligned}
& \frac{(r+1)^{\frac{-\alpha}{k}} t^{(r+1) \frac{\alpha}{k}}}{\Gamma_{k}(\alpha+k)} I_{a, k}^{\alpha, r} g^{2}(t)-\left[I_{a, k}^{\alpha, r} g(t)\right]^{2} \\
& \leq \quad\left(I_{a, k}^{\alpha, r} \psi_{2}(t)-I_{a, k}^{\alpha, r} g(t)\right)\left(I_{a, k}^{\alpha, r} g(t)-I_{a, k}^{\alpha, r} \psi_{1}(t)\right) \\
& \quad+\frac{(r+1)^{\frac{-\alpha}{k}} t^{(r+1) \frac{\alpha}{k}}}{\Gamma_{k}(\alpha+k)} I_{a, k}^{\alpha, r}\left(\psi_{1}(t) g(t)\right)-I_{a, k}^{\alpha, r} \psi_{1}(t) I_{a, k}^{\alpha, r} g(t) \\
& \quad+\frac{(r+1)^{\frac{-\alpha}{k}} t^{(r+1) \frac{\alpha}{k}}}{\Gamma_{k}(\alpha+k)} I_{a, k}^{\alpha, r}\left(\psi_{2}(t) g(t)\right)-I_{a, k}^{\alpha, r} \psi_{2}(t) I_{a, k}^{\alpha, r} g(t) \\
& \quad-\frac{(r+1)^{\frac{-\alpha}{k}} t^{(r+1) \frac{\alpha}{k}}}{\Gamma_{k}(\alpha+k)} I_{a, k}^{\alpha, r}\left(\psi_{1}(t) \psi_{2}(t)\right)+I_{a, k}^{\alpha, r} \psi_{1}(t) I_{a, k}^{\alpha, r} \psi_{2}(t) \\
& =S_{k}^{r}\left(g, \psi_{1}, \psi_{2}\right) .
\end{aligned}
$$

Equations (14) and (15) together with inequality (13) yield inequality (6). 
Lemma 2.11 Put $r=0$, the inequality (6) reduces to

$$
\left|\frac{t^{\frac{\alpha}{k}}}{\Gamma_{k}(\alpha+k)} I_{a, k}^{\alpha}(f(t) g(t))-I_{a, k}^{\alpha} f(t) I_{a, k}^{\alpha} g(t)\right| \leq \sqrt{S_{k}\left(f, \varphi_{1}, \varphi_{2}\right) S_{k}\left(f, \psi_{1}, \psi_{2}\right)},
$$

where

$$
\begin{aligned}
S_{k}(x, y, z)= & \left(I_{a, k}^{\alpha} z(t)-I_{a, k}^{\alpha} x(t)\right)\left(I_{a, k}^{\alpha} x(t)-I_{a, k}^{\alpha} y(t)\right) \\
& +\frac{t^{\frac{\alpha}{k}}}{\Gamma_{k}(\alpha+k)} I_{a, k}^{\alpha}(y(t) x(t))-I_{a, k}^{\alpha} y(t) I_{a, k}^{\alpha} x(t) \\
& +\frac{t^{\frac{\alpha}{k}}}{\Gamma_{k}(\alpha+k)} I_{a, k}^{\alpha}(z(t) x(t))-I_{a, k}^{\alpha} z(t) I_{a, k}^{\alpha} x(t) \\
& -\frac{t^{\frac{\alpha}{k}}}{\Gamma_{k}(\alpha+k)} I_{a, k}^{\alpha}(y(t) z(t))+I_{a, k}^{\alpha} y(t) I_{a, k}^{\alpha} z(t) .
\end{aligned}
$$

Example 2.12 For $k>0$, let $f$ and $g$ be two functions satisfying $t^{s} \leq f(t) \leq t^{s}+1$ and $t^{s}-1 \leq g(t) \leq t^{s}$ for $t \in[a, b]$. Then for $r \geq 0, \alpha>0$, we have

$$
\left|\frac{(r+1)^{\frac{-\alpha}{k}} t^{(r+1) \frac{\alpha}{k}}}{\Gamma_{k}(\alpha+k)} I_{a, k}^{\alpha, r}(f(t) g(t))-I_{a, k}^{\alpha, r} f(t) I_{a, k}^{\alpha, r} g(t)\right| \leq \sqrt{S_{k}^{r}\left(f, t^{s}, t^{s}+1\right) S_{k}^{r}\left(g, t^{s}-1, t^{s}\right)} .
$$

Here,

$$
\begin{aligned}
& S_{k}^{r}\left(f, t^{s}, t^{s}+1\right) \\
& =\left(\frac{(r+1)^{\frac{-\alpha}{k}} t^{(r+1) \frac{\alpha}{k}}}{\Gamma_{k}(\alpha+k)}+\frac{(r+1)^{\frac{-\alpha}{k}} t^{(r+1) \frac{\alpha}{k}+s} \Gamma_{k}\left(\frac{s k}{r+1}+k\right)}{\Gamma_{k}\left(\alpha+\frac{s k}{r+1}+k\right)}-I_{a, k}^{\alpha, r} f(t)\right) \\
& \times\left(I_{a, k}^{\alpha, r} f(t)-\frac{(r+1)^{\frac{-\alpha}{k}} t^{(r+1) \frac{\alpha}{k}+s} \Gamma_{k}\left(\frac{s k}{r+1}+k\right)}{\Gamma_{k}\left(\alpha+\frac{s k}{r+1}+k\right)}\right)+\frac{(r+1)^{\frac{-\alpha}{k}} t^{(r+1) \frac{\alpha}{k}}}{\Gamma_{k}(\alpha+k)} I_{a, k}^{\alpha, r}\left(f(t) t^{s}\right) \\
& -\frac{(r+1)^{\frac{-\alpha}{k}} t^{(r+1) \frac{\alpha}{k}+s} \Gamma_{k}\left(\frac{s k}{r+1}+k\right)}{\Gamma_{k}\left(\alpha+\frac{s k}{r+1}+k\right)} I_{a, k}^{\alpha, r} f(t)+\frac{(r+1)^{\frac{-\alpha}{k}} t^{(r+1) \frac{\alpha}{k}}}{\Gamma_{k}(\alpha+k)} I_{a, k}^{\alpha, r}\left(\left(t^{s}+1\right) f(t)\right) \\
& -\left(\frac{(r+1)^{\frac{-\alpha}{k}} t^{(r+1) \frac{\alpha}{k}+s} \Gamma_{k}\left(\frac{s k}{r+1}+k\right)}{\Gamma_{k}\left(\alpha+\frac{s k}{r+1}+k\right)}+\frac{(r+1)^{\frac{-\alpha}{k}} t^{(r+1) \frac{\alpha}{k}}}{\Gamma_{k}(\alpha+k)}\right) I_{a, k}^{\alpha, r} f(t) \\
& +\left(\frac{(r+1)^{\frac{-\alpha}{k}} t^{(r+1) \frac{\alpha}{k}+s} \Gamma_{k}\left(\frac{s k}{r+1}+k\right)}{\Gamma_{k}\left(\alpha+\frac{s k}{r+1}+k\right)}\right) \\
& \times\left(\frac{(r+1)^{\frac{-\alpha}{k}} t^{(r+1) \frac{\alpha}{k}+s} \Gamma_{k}\left(\frac{s k}{r+1}+k\right)}{\Gamma_{k}\left(\alpha+\frac{s k}{r+1}+k\right)}+\frac{(r+1)^{\frac{-\alpha}{k}} t^{(r+1) \frac{\alpha}{k}}}{\Gamma_{k}(\alpha+k)}\right) \\
& -\frac{(r+1)^{\frac{-\alpha}{k}} t^{(r+1) \frac{\alpha}{k}}}{\Gamma_{k}(\alpha+k)}\left(\frac{(r+1)^{\frac{-\alpha}{k}} t^{(r+1) \frac{\alpha}{k}+s} \Gamma_{k}\left(\frac{s k}{r+1}+k\right)}{\Gamma_{k}\left(\alpha+\frac{s k}{r+1}+k\right)}+\frac{(r+1)^{\frac{-\alpha}{k}} t^{(r+1) \frac{\alpha}{k}+2 s} \Gamma_{k}\left(\frac{2 s k}{r+1}+k\right)}{\Gamma_{k}\left(\alpha+\frac{2 s k}{r+1}+k\right)}\right),
\end{aligned}
$$

and

$$
\begin{aligned}
& S_{k}^{r}\left(f, t^{s}-1, t^{s}\right) \\
& \quad=\left(\frac{(r+1)^{\frac{-\alpha}{k}} t^{(r+1) \frac{\alpha}{k}}}{\Gamma_{k}(\alpha+k)}-\frac{(r+1)^{\frac{-\alpha}{k}} t^{(r+1) \frac{\alpha}{k}+s} \Gamma_{k}\left(\frac{s k}{r+1}+k\right)}{\Gamma_{k}\left(\alpha+\frac{s k}{r+1}+k\right)}+I_{a, k}^{\alpha, r} g(t)\right)
\end{aligned}
$$




$$
\begin{aligned}
& \times\left(\frac{(r+1)^{\frac{-\alpha}{k}} t^{(r+1) \frac{\alpha}{k}+s} \Gamma_{k}\left(\frac{s k}{r+1}+k\right)}{\Gamma_{k}\left(\alpha+\frac{s k}{r+1}+k\right)}-I_{a, k}^{\alpha, r} g(t)\right)+\frac{(r+1)^{\frac{-\alpha}{k}} t^{(r+1) \frac{\alpha}{k}}}{\Gamma_{k}(\alpha+k)} I_{a, k}^{\alpha, r}\left(\left(t^{s}-1\right) g(t)\right) \\
& -\left(\frac{(r+1)^{\frac{-\alpha}{k}} t^{(r+1) \frac{\alpha}{k}+s} \Gamma_{k}\left(\frac{s k}{r+1}+k\right)}{\Gamma_{k}\left(\alpha+\frac{s k}{r+1}+k\right)}-\frac{(r+1)^{\frac{-\alpha}{k}} t^{(r+1) \frac{\alpha}{k}}}{\Gamma_{k}(\alpha+k)}\right) I_{a, k}^{\alpha, r} g(t) \\
& +\frac{(r+1)^{\frac{-\alpha}{k}} t^{(r+1) \frac{\alpha}{k}}}{\Gamma_{k}(\alpha+k)} I_{a, k}^{\alpha, r}\left(t^{s} g(t)\right)-\frac{(r+1)^{\frac{-\alpha}{k}} t^{(r+1) \frac{\alpha}{k}+s} \Gamma_{k}\left(\frac{s k}{r+1}+k\right)}{\Gamma_{k}\left(\alpha+\frac{s k}{r+1}+k\right)} I_{a, k}^{\alpha, r} g(t) \\
& +\left(\frac{(r+1)^{\frac{-\alpha}{k}} t^{(r+1) \frac{\alpha}{k}+s} \Gamma_{k}\left(\frac{s k}{r+1}+k\right)}{\Gamma_{k}\left(\alpha+\frac{s k}{r+1}+k\right)}-\frac{(r+1)^{\frac{-\alpha}{k}} t^{(r+1) \frac{\alpha}{k}}}{\Gamma_{k}(\alpha+k)}\right) \\
& \times\left(\frac{(r+1)^{\frac{-\alpha}{k}} t^{(r+1) \frac{\alpha}{k}+s} \Gamma_{k}\left(\frac{s k}{r+1}+k\right)}{\Gamma_{k}\left(\alpha+\frac{s k}{r+1}+k\right)}\right) \\
& -\frac{(r+1)^{\frac{-\alpha}{k}} t^{(r+1) \frac{\alpha}{k}}}{\Gamma_{k}(\alpha+k)}\left(\frac{(r+1)^{\frac{-\alpha}{k}} t^{(r+1) \frac{\alpha}{k}+s} \Gamma_{k}\left(\frac{s k}{r+1}+k\right)}{\Gamma_{k}\left(\alpha+\frac{s k}{r+1}+k\right)}-\frac{(r+1)^{\frac{-\alpha}{k}} t^{(r+1) \frac{\alpha}{k}+2 s} \Gamma_{k}\left(\frac{2 s k}{r+1}+k\right)}{\Gamma_{k}\left(\alpha+\frac{2 s k}{r+1}+k\right)}\right) .
\end{aligned}
$$

Conclusions The present research is the generalizations and extensions of Grüss type inequalities in the form of new symbol $k>0$. Finally, if take $k=1$, we have the classical Grüss type inequalities.

\section{Competing interests}

The authors declare that they have no competing interests.

\section{Authors' contributions}

The authors SM and SI contributed and approved equally to the writing of this paper.

\section{Acknowledgements}

The authors would like to express profound gratitude to referees and the editor for deeper review of this paper and their valuable advice. The authors are also pleased to pay special thanks to Dr. Mumtaz Ahmad for his support in this research.

Received: 8 October 2015 Accepted: 22 March 2016 Published online: 04 April 2016

\section{References}

1. Dragomir, SS: A generalization of Grüss inequality in inner product spaces and applications. J. Math. Anal. Appl. 237 74-82 (1999)

2. Elezovic, N, Marangunic, LJ, Pecaric, J: Some improvements of Grüss type inequality. J. Math. Inequal. 1, 425-436 (2007)

3. Li, X, Mohapatra, RN, Rodriguez, RS: Grüss-type inequalities. J. Math. Anal. Appl. 267, 434-443 (2002)

4. Pachpatte, BG: On multidimensional Grüss type inequalities. J. Inequal. Pure Appl. Math. 3, 1-15 (2002)

5. Wang, G, Agarwal, P, Chand, M: Certain Grüss type inequalities involving the generalized fractional integral operator. J. Inequal. Appl. 2014, Article ID 147 (2014)

6. Kaçar, E, Yildirim, H: Grüss type integral inequalities for generalized Riemann-Liouville fractional integrals. Int. J. Pure Appl. Math. 101(1), 55-70 (2015)

7. Akin, E, Asliyüce, S, Güvenilir, AF, Kaymakçalan, B: Discrete Grüss type inequality on fractional calculus. J. Inequal. Appl. 2015, Article ID 174 (2015)

8. Grüss, G: Uber das maximum des absoluten Betrages $\frac{1}{b-a} \int_{a}^{b} f(x) g(x) d x-\frac{1}{(b-a)^{2}} \int_{a}^{b} f(x) d x \frac{1}{b-a} \int_{a}^{b} g(x) d x$. Math. Z. 39, 215-226 (1935)

9. Tariboon, J, Ntouyas, SK, Sudsutad, W: Some new Riemann-Liouville fractional integral inequalities. Int. J. Math. Math. Sci. 2014, Article ID 869434 (2014)

10. Sarikaya, MZ, Filiz, H, Kiris, ME: On some generalized integral inequalities for Riemann-Liouville fractional integrals. Filomat 29(6), 1307-1314 (2015)

11. Dahmani, Z: About some integral inequalities using Riemann-Liouville integrals. Gen. Math. 20(4), 63-69 (2012)

12. Liao, Y, Deng, J, Wang, J: Riemann-Liouville fractional Hermite-Hadamard inequalities. Part I: for once differentiable geometric-arithmetically s-convex functions. J. Inequal. Appl. 2013, Article ID 443 (2013)

13. Chen, F: A note on Hermite-Hadamard inequalities for products of convex functions via Riemann-Liouville fractional integrals. Ital. J. Pure Appl. Math. 2014(33), 299-306 (2014)

14. Park, J: Some integral inequalities for convex functions via Riemann-Liouville integrals. Appl. Math. Sci. 9(27), 1341-1353 (2015)

15. Tunc, M: On new inequalities for $h$-convex functions via Riemann-Liouville fractional integration. Filomat 27(4), 559-565 (2013)

16. Diaz, R, Pariguan, E: On hypergeometric functions and Pochhammer k-symbol. Divulg. Mat. 15, 179-192 (2007) 
17. Krasniqi, $\mathrm{V}$ : Inequalities and monotonicity for the ration of $k$-gamma functions. Sci. Magna 6(1), 40-45 (2010)

18. Mubeen, S, Habibullah, GM: k-Fractional integrals and application. Int. J. Contemp. Math. Sci. 7, 89-94 (2012)

19. Kokologiannaki, CG, Krasniqi, V: Some properties of the k-gamma function. Matematiche 68(1), 13-22 (2013)

20. Romero, LG, Luque, LL, Dorrego, GA, Cerutti, RA: On the k-Riemann-Liouville fractional derivative. Int. J. Contemp. Math. Sci. 8(1), 41-51 (2013)

21. Baleanu, D, Agarwal, P: Certain inequalities involving the fractional $q$-integral operators. Abstr. Appl. Anal. 2014 Article ID 371274 (2014)

22. Choi, J, Agarwal, P: Some new Saigo type fractional integral inequalities and their $q$-analogues. Abstr. Appl. Anal. 2014, Article ID 579260 (2014)

23. Agarwal, P, Salahshour, S, Ntouyas, SK, Tariboon, J: Certain inequalities involving generalized Erdélyi-Kober fractional q-integral operators. Sci. World J. 2014, Article ID 174126 (2014)

24. Sarikaya, MZ, Karaca, A: On the k-Riemann-Liouville fractional integral and applications. Int. J. Stat. Math. 1(3), 33-43 (2014)

25. Choi, J, Ritelli, D, Agarwal, P: Some new inequalities involving generalized Erdélyi-Kober fractional $q$-integral operator. Appl. Math. Sci. 9(72), 3577-3591 (2015)

26. Liu, X, Zhang, L, Agarwal, P, Wang, G: On some new integral inequalities of Gronwall-Bellman-Bihari type with delay for discontinuous functions and their applications. Indag. Math. 27(1), 1-10 (2016). doi:10.1016/j.indag.2015.07.00

27. Sarikaya, MZ, Dahmani, Z, Kiris, ME, Ahmad, F: (k, s)-Riemann-Liouville fractional integral and applications. Hacet. J. Math. Stat. 45(1), 77-89 (2016)

\section{Submit your manuscript to a SpringerOpen ${ }^{\ominus}$ journal and benefit from:}

- Convenient online submission

- Rigorous peer review

- Immediate publication on acceptance

- Open access: articles freely available online

- High visibility within the field

- Retaining the copyright to your article 